\title{
HLA-DRB1 Haplotypes, Shared Epitope, and Disease Outcomes in US Veterans with Rheumatoid Arthritis
}

\author{
Ming Zhao, Lilli Mauer, Harlan Sayles, Grant W. Cannon, Andreas Reimold, Gail S. Kerr, \\ Joshua F. Baker, Geoffrey M. Thiele, Bryant R. England (1), and Ted R. Mikuls
}

ABSTRACT. Objective. To evaluate associations of HLA-DRB1 haplotypes and shared epitope (SE) with rheumatoid arthritis (RA) severity and all-cause mortality in RA.

Methods. Patients with RA from the Veterans Affairs Rheumatoid Arthritis (VARA) registry were followed from enrollment until death or December 31, 2013. Clinical characteristics, DNA, and serum were collected at enrollment. Radiographic damage, the presence or absence of subcutaneous nodules, disease activity measures, and functional status were assessed at enrollment and updated during followup. Sixteen HLA-DRB1 haplotypes and SE status were determined from banked DNA. Associations between HLA-DRB1 haplotypes, RA disease characteristics, and mortality were assessed in multivariable regression models.

Results. Within VARA, 1443 participants had genotyping and accrued 6150 patient-years of followup. Haplotypes VKA, VRA, LRA, SRA, SRE, SKR, and SEA, and SE alleles were significantly associated with seropositivity for rheumatoid factor (RF) and/or anticyclic citrullinated peptide (anti-CCP). Haplotypes VKA and SKR were associated with higher RF concentrations, while VRA, DRE, and GRQ were associated with lower RF concentrations. Haplotypes VKA, VRA, and LRA were associated with higher concentrations of anti-CCP antibody, while haplotypes SRA, SRE, LEA, SKR, and SEA were significantly associated with lower anti-CCP concentrations. Haplotype VKA (OR $1.39,95 \%$ CI 1.08-1.80) was associated with increased frequency of radiographic damage at enrollment but none of the haplotypes were associated with the presence of subcutaneous nodules. Haplotypes SKA (HR 1.52, 95\% CI 1.26-1.83) was associated with higher mortality.

Conclusion. HLA-DRB1 haplotypes are independently and variably associated with seropositivity, autoantibody concentrations, and outcomes in RA. (First Release March 1 2019; J Rheumatol 2019;46:685-93; doi:10.3899/jrheum.180724)

Key Indexing Terms:

RHEUMATOID ARTHRITIS HLA-DRB1 HAPLOTYPES RHEUMATOID FACTOR ANTICYCLIC CITRULLINATED PEPTIDE RADIOGRAPHIC DAMAGE MORTALITY

\footnotetext{
From the Veterans Affairs (VA) Nebraska-Iowa Health Care System; Division of Rheumatology and Immunology, Department of Internal Medicine, University of Nebraska Medical Center (UNMC), Omaha, Nebraska, USA.

Funding provided by UNMC Internal Medicine Scientist Development Award, UNMC Physician-Scientist Training Program, UNMC Mentored Scholars Program, US National Institute of General Medical Sciences (U54GM115458), National Institute on Alcohol Abuse and Alcoholism (R25AA020818), and the National Institute of Arthritis and Musculoskeletal and Skin Diseases (2P50AR60772).

M. Zhao, MD, VA Nebraska-Iowa Healthcare System, and Division of Rheumatology and Immunology, Department of Internal Medicine, UNMC; L. Mauer, MD, VA Nebraska-Iowa Healthcare System, and Division of Rheumatology and Immunology, Department of Internal Medicine, UNMC; H. Sayles, MS, Division of Rheumatology and Immunology, Department of Internal Medicine, UNMC, and Department of Biostatistics, College of Public Health, UNMC; G.W. Cannon, MD, VA Salt Lake City and University of Utah; A. Reimold, MD, Dallas VA and University of Texas Southwestern; G.S. Kerr, MD, Washington DC VAMC, Georgetown University and Howard University; J.F. Baker, MD, MSCE, Corporal Michael J. Crescenz VA and University of Pennsylvania; G.M. Thiele, PhD, VA Nebraska-Iowa Healthcare System, and Division of Rheumatology and Immunology, Department of Internal Medicine, UNMC; B.R. England, MD, VA Nebraska-Iowa Healthcare System, and Division of Rheumatology and Immunology, Department of Internal Medicine, UNMC; T.R. Mikuls, MD, MSPH, VA Nebraska-Iowa
}

Healthcare System, and Division of Rheumatology and Immunology, Department of Internal Medicine, UNMC.

Address correspondence to Dr. T.R. Mikuls, University of Nebraska Medical Center, 986270 Nebraska Medical Center, Omaha, Nebraska 68198-6270,USA.E-mail:tmikuls@unmc.edu,tmikuls@yahoo.com Accepted for publication October 30, 2018.

Numerous genetic susceptibility loci have been identified in rheumatoid arthritis (RA). The HLA-DRB1 shared epitope (SE) sequence, a motif encompassing amino acids 70 through 74 , has traditionally been recognized as exerting the greatest heritable influence in disease susceptibility ${ }^{1,2,3,4,5}$. In contrast to its more established links with disease susceptibility, the potential relationship of HLA-DRB1 SE alleles with disease phenotype and outcome has been less clear. Studies, for example, investigating the association of SE status with RA-related mortality have yielded inconsistent results. While some reports have demonstrated associations of SE status with premature mortality, particularly from cardiovascular disease ${ }^{3,6,7}$, others failed to replicate these findings after accounting for relevant confounding factors ${ }^{8,9}$.

Personal non-commercial use only. The Journal of Rheumatology Copyright (C) 2019. All rights reserved. 
In addition to its proposed relationship with survival, the HLA-DRB1 SE allele has been linked to seropositivity to both rheumatoid factor (RF) and anticyclic citrullinated peptide (anti-CCP), which is important given the relationship of these antibodies with RA disease progression ${ }^{4,10,11}$. Possibly mediated by autoantibody expression ${ }^{12}$, inheritance of the SE allele has also been associated with a higher incidence of radiographic erosions ${ }^{13,14}$ as well as the expression of select extraarticular disease manifestations ${ }^{15}$. Although studies have reported a relationship between HLA-DRB1 SE positivity and the presence of rheumatoid nodules ${ }^{5}$, the most common extraarticular disease manifestation, a metaanalysis involving more than 3200 patients failed to demonstrate these findings, with the exception of a weak relationship between inheritance of a single DRB $1 * 0401$ allele ${ }^{16}$.

Recently, there has been increasing interest in amino acids that also comprise HLA-DRB1 but fall outside the immediate SE region. Specifically, investigators have identified 16 HLA-DRB1 haplotypes defined by amino acids at positions 11,71 , and 74 that appear to better predict RA risk and disease outcomes than SE status alone, understanding the existence of significant overlap between SE status and the newly described haplotypes ${ }^{17,18}$. Valine at position 11 , commonly observed in conjunction with select SE alleles, appears to be among the most robust genetic predictors of disease risk ${ }^{17,19}$. It has been proposed that valine in this position, located at the base of the HLA-DR peptide binding groove, facilitates autoantigen binding ${ }^{18}$. In addition to a role in disease susceptibility, Viatte and colleagues examined the relationship of these 16 HLA-DRB1 haplotypes with disease outcomes, demonstrating strong associations between valine at position 11 and its related haplotypes (particularly the VKA haplotype) with all-cause mortality, the presence of radiographic erosions, and improved treatment response in patients with $\mathrm{RA}^{17}$.

In this study of United States veterans with RA, we sought to externally validate these previous findings by evaluating the associations of these 16 HLA-DRB1 haplotypes, along with the SE risk alleles, with all-cause mortality and the presence of radiographic damage. In addition, we also examined the relationship of these genetic susceptibility factors with the presence of subcutaneous nodules, $\mathrm{RF}$, and anti-CCP antibody status. We hypothesized that HLA-DRB1 haplotypes associated with predisposition to RA would also be associated with clinical markers of severity such as radiographic damage, nodules, disease activity, and all-cause mortality, and would be more closely associated with anti-CCP antibody rather than RF.

\section{MATERIALS AND METHODS}

Study population. The study population included participants from the Veterans Affairs Rheumatoid Arthritis (VARA) registry, a multicentered longitudinal observational study begun in 2003 of US veterans with RA ${ }^{20}$. For this study, patients were enrolled from rheumatology clinics at VA medical centers in the following 12 US cities: Birmingham, Alabama; Brooklyn, New York; Dallas, Texas; Denver, Colorado; Iowa City, Iowa; Jackson, Mississippi; Little Rock, Arkansas; Omaha, Nebraska; Philadelphia, Pennsylvania; Portland, Oregon; Salt Lake City, Utah; and Washington, DC. VARA was approved by the VA Nebraska-Western Iowa Health Care System Institutional Review Board (IRB; Protocol no. 00149) in addition to receiving IRB approval from the other 11 aforementioned participating sites. All participants provided written informed consent prior to enrollment and satisfied the 1987 American College of Rheumatology (ACR) classification criteria ${ }^{21}$.

Clinical assessments and vital status. At enrollment, the following information was collected: age, sex, ancestry (race/ethnicity), smoking status (current, former, never), body mass index (BMI), medications, educational status, comorbid conditions, and date of RA diagnosis. The Rheumatic Diseases Comorbidity Index (RDCI) was examined as a measure of overall comorbidity burden ${ }^{22}$. DNA and serum were collected and banked at the time of enrollment. Using banked serum, anti-CCP was measured using second-generation ELISA (Diastat; Axis-Shield Diagnostics, positivity $\geq 5$ $\mathrm{U} / \mathrm{ml}$ ), and RF was measured by nephelometry (Siemens; positivity $\geq 15$ $\mathrm{IU} / \mathrm{ml})^{23}$. At enrollment and followup visits conducted as part of routine rheumatology care, the following were collected: swollen and/or tender joint counts ( $0-28$ joints), patient-reported pain (10-point scale), patient-reported global disease assessment (100-mm scale), physician-reported global disease assessment (100-mm scale), Multidimensional Health Assessment Questionnaire (scale $0-3$ points), erythrocyte sedimentation rate $(\mathrm{mm} / \mathrm{h})$, and C-reactive protein $(\mathrm{mg} / \mathrm{dl})$ level. ACR classification criteria, including evidence of radiographic damage and the presence or absence of subcutaneous nodules are systematically assessed only at enrollment. For this study, participants were followed from enrollment until death or December 31 , 2013, whichever occurred first. Vital status and date of death were ascertained through linkage to the National Death Index (Center for Excellence for Suicide Prevention, Joint Department of VA and Department of Defense Suicide Data Repository; vaww.virec.research.va.gov/Mortality/Overview. htm; extracted through 2013) ${ }^{9}$. Cause of death was classified as cardiovascular by the presence of International Classification of Diseases, 10th revision, Chapter IX codes I00-I99.

Genotyping. Using banked DNA, 4-digit HLA-DRB1 genotypes and SE status were determined as previously described on a subset of registry participants, using 1 of 2 approaches: DNA sequencing of exon 2 using the AlleleSEQR HLA-DRB1 reagent kit and protocol (Abbott Molecular) or with a PCR-based, sequence-specific oligonucleotide probe system ${ }^{24}$. Using the 4-digit genotypes, amino acids at HLA-DRB1 positions 11, 71, and 74 were assigned using the IMGT/HLA Database (European Bioinformatics Institute; www.ebi.ac.uk/ipd/imgt/hla) and haplotypes generated as described by Viatte, et al ${ }^{17}$.

Statistical analysis. Associations between HLA-DRB1 haplotypes with select medications [methotrexate (MTX), prednisone, biologics], RF, and anti-CCP positivity were assessed with chi-square tests. RF and anti-CCP concentrations were log transformed for normality and compared between individuals with and without HLA-DRB1 haplotypes, as well as SE alleles, using independent $t$ tests. Because concentrations of autoantibodies are most informative in those with seropositivity, we additionally compared autoantibody concentrations between haplotypes among those who were seropositive. Cross-sectional associations with clinical sequelae of RA, subcutaneous nodules, and radiographic changes were estimated using multivariable logistic regression, adjusting for sex, race, smoking status, and disease duration. These were limited to cross-sectional analyses as these outcomes are systematically assessed only at the time of registry enrollment. Associations of haplotypes with longitudinal disease activity were assessed using generalized estimating equations (GEE) with varying levels of covariate adjustment. A bare model included age, sex, race, smoking status, RDCI score, duration of followup, HLA-DRB1 haplotypes, and the interaction of haplotypes with time. A fully adjusted model also included variables potentially residing in the causal pathway (autoantibody status,

Personal non-commercial use only. The Journal of Rheumatology Copyright $\odot$ (2019. All rights reserved 
MTX use, biologic disease-modifying antirheumatic drug use, and prednisone use). We tested interactions between sex, race, autoantibody positivity, current smoking, and RDCI score with time. These terms were not significant $(\beta<0.05$ and $p$ value $>0.10)$ and thus not included in the final multivariable models. An unstructured covariance structure produced the model with the best chi-square value and was used in all GEE models.

Mortality data were analyzed using Cox proportional hazards regression models. Two models were constructed with varying levels of adjustment and excluding factors that could be in the causal pathway (e.g., disease activity): (1) age, sex, and race; (2) age, sex, race, smoking status, and RDCI. The covariates included were chosen based on prior studies of the VARA cohort $^{9,20}$. In multivariable models, we compared subjects possessing at least 1 copy of the haplotype under investigation to those with no copies.

To examine whether any null findings observed for mortality and nodules might be related to simultaneous influence of other haplotypes or due to underlying ancestry, additional sensitivity analyses were conducted. Specifically, we performed additional analyses examining haplotype associations with nodules and all-cause mortality with each allele as the primary unit, rather than the patient. This allowed us to compare each HLA-DRB1 haplotype referent to the PAA haplotype regardless of the haplotype present on the other allele. To account for correlated data introduced with this approach, all regression models incorporated robust standard errors and were clustered on the patient. Given that HLA-DRB1 risk alleles vary by ancestry, we also performed additional analyses examining these same outcomes that were limited to white patients.

$\mathrm{P}$ values $<0.05$ were considered statistically significant and all analyses were completed using Stata version 14.1 (StataCorp).

\section{RESULTS}

Baseline characteristics. A total of 1443 participants with available genotyping data were studied with 6150 patient-years of followup. A majority of the subjects were white (79.3\%) and male (91.2\%) with a mean (SD) age of 64 years (11). SE alleles were present in $71.7 \%$ of participants. Most were seropositive for RF (80.8\%) or anti-CCP antibody (78.6\%). Detailed baseline characteristics are shown in Table 1.

Association with seropositivity and autoantibody concentrations. VKA was the most common haplotype observed, with $34 \%$ of patients possessing at least 1 copy (Table 2). Haplotypes VKA, VRA, LRA, and presence of SE alleles were significantly more frequent with seropositivity (all $\mathrm{p}$ values $\leq 0.02$ ), while the SRA, SRE, SKR, and SEA haplotypes were significantly more likely to accompany seronegative status (all $p$ values $\leq 0.02$ ). Notably, the associations of VKA, VRA, and LRA with autoantibody positivity were more closely linked to anti-CCP antibody positivity than RF positivity. A similar pattern was observed for SE positivity. Specifically, the frequency of SE positivity was nearly identical in those positive for both RF and anti-CCP (76.6\%) to those positive for only anti-CCP $(78.7 \%)$, with lower frequencies observed in both seronegative patients $(55.6 \%)$ or those positive for only RF $(53.1 \%$; p value $<0.001)$.

Associations of haplotype status with autoantibody concentrations are shown in Table 3. Haplotypes VKA (347 vs $321 \mathrm{IU} / \mathrm{ml}, \mathrm{p}<0.001)$ and SKR (342 vs $327 \mathrm{IU} / \mathrm{ml}$, $\mathrm{p}=0.05)$ were associated with higher RF concentrations, while VRA (317 vs $334 \mathrm{IU} / \mathrm{ml}, \mathrm{p}=0.05$ ), DRE (294 vs 331 $\mathrm{IU} / \mathrm{ml}, \mathrm{p}=0.05)$, and GRQ (218 vs $352 \mathrm{IU} / \mathrm{ml}, \mathrm{p}=0.02)$ were
Table 1. Baseline characteristics.

\begin{tabular}{|c|c|}
\hline Characteristics & Values \\
\hline $\mathrm{N}$ & 1443 \\
\hline Age, yrs & $64(11)$ \\
\hline White, \% & 79.3 \\
\hline Male, $\%$ & 91.2 \\
\hline \multicolumn{2}{|l|}{ Smoking, \% } \\
\hline Current & 27.0 \\
\hline Former & 52.5 \\
\hline Never & 20.4 \\
\hline \multicolumn{2}{|l|}{ BMI group, $\mathrm{kg} / \mathrm{m}^{2}, \%$} \\
\hline$<20$ (underweight) & 4.4 \\
\hline 20 to $<25$ (normal) & 23.3 \\
\hline 25 to $<30$ (overweight) & 36.1 \\
\hline 30 to $<35$ (obese) & 20.7 \\
\hline$\geq 35$ (morbidly obese) & 10.0 \\
\hline RDCI (0-9) & $2.3(1.7)$ \\
\hline Shared epitope-positive, $\%$ & 71.7 \\
\hline RF-positive, $\%$ & 80.8 \\
\hline Anti-CCP-positive, $\%$ & 78.6 \\
\hline Nodules, $\%$ & 33.3 \\
\hline MDHAQ (0-3) & $0.9(0.6)$ \\
\hline \multicolumn{2}{|l|}{ DAS28 state, $\%$} \\
\hline Remission & 22.5 \\
\hline Low & 14.6 \\
\hline Moderate & 39.2 \\
\hline High & 22.9 \\
\hline \multicolumn{2}{|l|}{ DMARD, $\%$} \\
\hline None & 13.7 \\
\hline Non-MTX & 20.4 \\
\hline MTX & 38.3 \\
\hline Biologic & 27.7 \\
\hline Prednisone, $\%$ & 40.3 \\
\hline
\end{tabular}

Values are mean (SD) unless otherwise noted; cumulative followup of 6150 patient-years. Data are missing for prednisone use (24 subjects), DAS (12), BMI group (80). BMI: body mass index; RDCI: Rheumatic Diseases Comorbidity Index; RF: rheumatoid factor; CCP: cyclic citrullinated peptides; MDHAQ: Multidimensional Health Assessment Questionnaire; DAS28: 28-joint count Disease Activity Score; DMARD: disease-modifying antirheumatic drug; MTX: methotrexate.

associated with lower RF concentrations. When restricting these analyses to RF-positive individuals, only the association between GRQ and lower RF concentrations remained significant. SE positivity was associated with higher concentration of RF overall (337 vs $311 \mathrm{IU} / \mathrm{ml}, \mathrm{p}<0.001$ ) and in RF-positive patients (420 vs $408 \mathrm{IU} / \mathrm{ml}, \mathrm{p}=0.008$ ).

Haplotypes VKA (331 vs $243 \mathrm{U} / \mathrm{ml}, \mathrm{p}<0.001)$, VRA (303 vs $261 \mathrm{U} / \mathrm{ml}, \mathrm{p}<0.001$ ), and LRA (311 vs $259 \mathrm{U} / \mathrm{ml}$, $\mathrm{p}=0.02)$ were associated with higher concentrations of anti-CCP antibody in all patients, but only LRA (389 vs 340 $\mathrm{U} / \mathrm{ml}, \mathrm{p}=0.03$ ) was found to have the same association in anti-CCP-positive patients. These haplotypes were significantly associated with lower anti-CCP concentration in all patients: SRA (244 vs $277 \mathrm{U} / \mathrm{ml}, \mathrm{p}=0.01$ ), SRE (211 vs 275 $\mathrm{U} / \mathrm{ml}, \mathrm{p}=0.006)$, LEA (126 vs $275 \mathrm{U} / \mathrm{ml}, \mathrm{p}=0.02)$, SKR (155 vs $296 \mathrm{U} / \mathrm{ml}, \mathrm{p}<0.001)$, and SEA (191 vs $287 \mathrm{U} / \mathrm{ml}, \mathrm{p}<$ 0.001). SKR (259 vs $367 \mathrm{U} / \mathrm{ml}, \mathrm{p}<0.001)$ and SEA (284 vs

Personal non-commercial use only. The Journal of Rheumatology Copyright $\subset$ 2019. All rights reserved. 
Table 2. Frequency of HLA-DRB1 SE and haplotype status (1 or more copy) based on dual RF and anti-CCP antibody status.

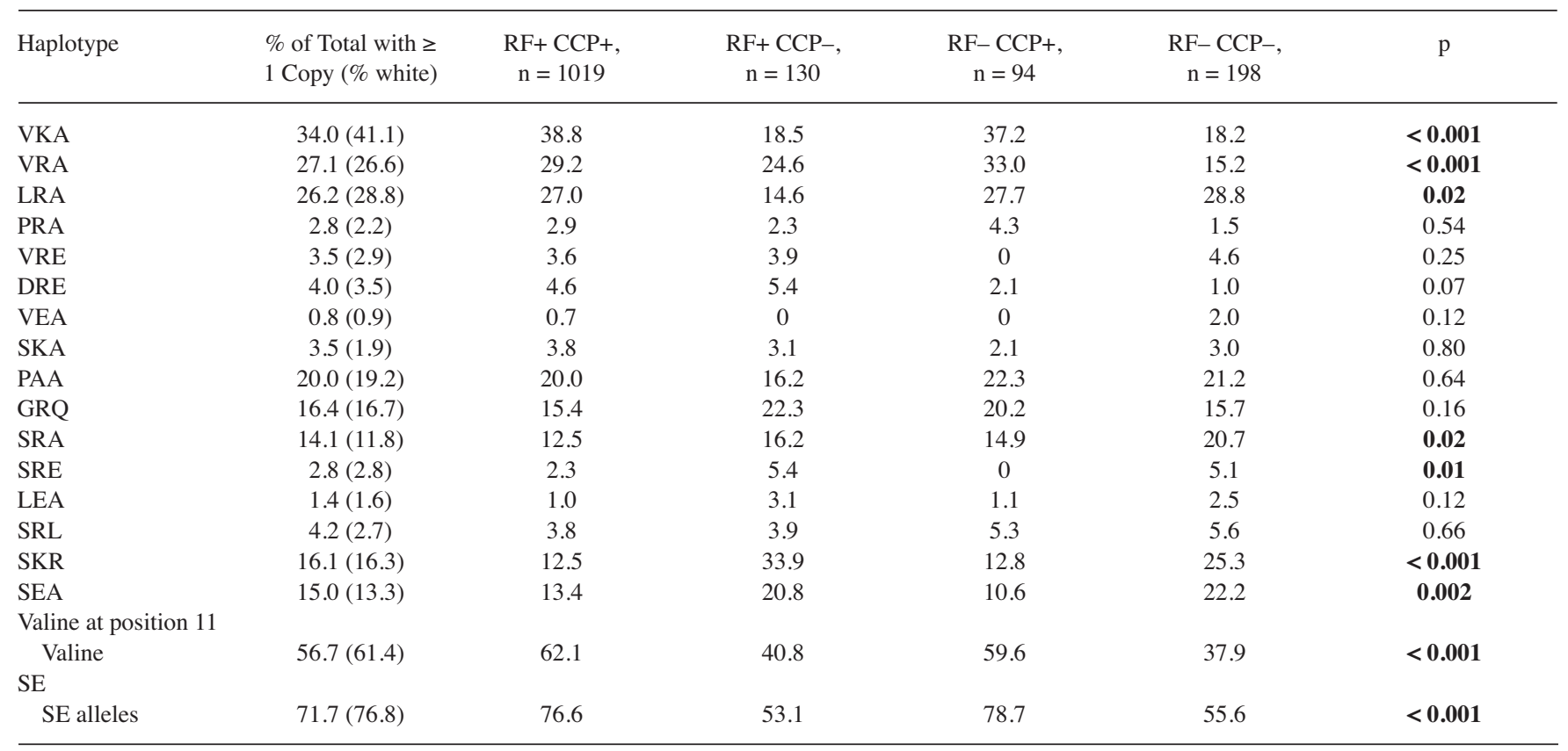

P value by chi-square. P values in bold face are statistically significant. RF: rheumatoid factor; CCP: cyclic citrullinated peptides; SE: shared epitope.

$364 \mathrm{U} / \mathrm{ml}, \mathrm{p}=0.003$ ) were associated with lower anti-CCP antibody concentrations among anti-CCP-positive patients. Presence of SE alleles was associated with higher concentration of anti-CCP overall (310 vs $178 \mathrm{U} / \mathrm{ml}, \mathrm{p}<0.001)$ and anti-CCP-positive patients (375 vs $281 \mathrm{U} / \mathrm{ml}, \mathrm{p}<0.001$ ). Stratified by smoking status, the associations between HLA-DRB1 haplotypes with RF concentrations were attenuated (Supplementary Table 1, available with the online version of this article). VKA and SE remained associated with higher anti-CCP antibody concentrations, while SKR and SEA remained associated with lower anti-CCP antibody concentrations after stratifying by smoking status (Supplementary Table 2).

Association with subcutaneous nodules, radiographic damage, and disease activity. None of the HLA-DRB1 haplotypes were significantly associated with the presence of subcutaneous nodules (Table 4). These null findings were replicated using an allele-based approach that simultaneously accounted for the effects of different haplotypes with comparisons referent to the PAA haplotype (all $\mathrm{p}$ values $\geq 0.14$, data not shown). Likewise, these results were unchanged when analyses were restricted to whites (all $\mathrm{p}$ values $>0.11$, data not shown).

Haplotype VKA (OR 1.39, 95\% CI 1.08-1.80) was associated with an increased frequency of radiographic damage. Combining all the haplotypes with valine at position 11 (VKA, VRA, VRE, and VEA), we found a significant association with radiographic changes (OR 1.31, 95\% CI 1.04-1.67). These results were unchanged in analyses restricted to whites (data not shown). In analyses of the entire study population, SE positivity was marginally associated with both nodules (OR 1.25, 95\% CI 0.96-1.63) and radiographic changes (OR 1.23, 95\% CI 0.95-1.60), neither of which reached statistical significance.

In GEE models, haplotypes DRE and VEA were associated with increased disease activity at enrollment, while haplotypes SKA and LEA were associated with a modest increased disease activity over time (Supplementary Table 3, available with the online version of this article). Results were similar in bare and fully adjusted models. In separate analyses examining haplotype associations with baseline treatments that might be indicative of more severe disease, we found no associations of any of the haplotypes with MTX use (Supplementary Table 4). In contrast, VKA $(32 \%$ vs $26 \%, p=0.03)$, $\operatorname{VRE}(43 \%$ vs $28 \%, p=0.02)$, and haplotypes with valine at position $11(30 \%$ vs $25 \%, \mathrm{p}=0.02)$ were associated with a higher frequency of biologic use. Of the haplotypes examined, only SKA was associated with greater prednisone use $(60 \%$ vs $42 \%, \mathrm{p}=0.01)$,

Association with mortality. Haplotypes SKA (HR 1.52, 95\% CI 1.26-1.83) was associated with higher mortality in analysis adjusted for age, sex, and race (Figure 1). Further adjustment for smoking status and RDCI score did not significantly attenuate this association (SKA HR 1.57, 95\% CI 1.25-1.98). There were trends toward improved survival with VRE (HR 0.67,95\% CI 0.39-1.14) and LEA (HR 0.79, 95\% CI 0.42-1.48) haplotypes; however, the estimates were imprecise and not statistically significant. The remaining haplotypes, including VKA, were not significantly associated with mortality. Neither the presence of SE (HR $0.92,95 \%$ CI $0.71-1.19)$ nor valine at position 11 (HR $0.96,95 \% \mathrm{CI}$

Personal non-commercial use only. The Journal of Rheumatology Copyright $\subset$ $\subset$ 2019. All rights reserved 
Table 3A. Mean autoantibody (RF) concentrations across haplotype groups and by HLA-DRB1 status.

\begin{tabular}{|c|c|c|c|c|c|c|}
\hline Haplotype & Haplotype+ & $\begin{array}{c}\text { All, } n=1442 \\
\text { Haplotype- }\end{array}$ & $\mathrm{p}^{*}$ & Haplotype+ & $\begin{array}{c}\mathrm{RF}+, \mathrm{n}=1150 \\
\text { Haplotype- }\end{array}$ & $\mathrm{p}$ \\
\hline VKA & 347 (639) & $321(676)$ & $<0.001$ & $404(675)$ & 415 (746) & 0.27 \\
\hline VRA & $317(520)$ & $334(709)$ & 0.05 & $374(548)$ & $426(779)$ & 0.93 \\
\hline LRA & $356(766)$ & $321(623)$ & 0.95 & $453(843)$ & $396(673)$ & 0.30 \\
\hline PRA & $231(579)$ & $333(665)$ & 0.46 & $278(629)$ & 415 (723) & 0.13 \\
\hline VRE & $242(341)$ & $333(672)$ & 0.96 & $292(357)$ & $415(730)$ & 0.65 \\
\hline DRE & $294(401)$ & $331(672)$ & 0.05 & 315 (407) & $416(732)$ & 0.76 \\
\hline VEA & $298(635)$ & $330(664)$ & 0.50 & $462(766)$ & $411(721)$ & 0.79 \\
\hline SKA & $293(522)$ & $331(668)$ & 0.98 & 345 (554) & $413(726)$ & 0.46 \\
\hline PAA & $359(734)$ & $322(645)$ & 0.66 & $457(803)$ & 400 (699) & 0.89 \\
\hline GRQ & $218(371)$ & $352(705)$ & 0.02 & $273(400)$ & $438(765)$ & 0.002 \\
\hline SRA & 359 (649) & 325 (666) & 0.47 & 489 (719) & $399(720)$ & 0.10 \\
\hline SRE & 415 (968) & 327 (653) & 0.41 & 550 (1089) & 407 (708) & 0.72 \\
\hline LEA & $170(282)$ & 332 (667) & 0.09 & $239(315)$ & 413 (724) & 0.19 \\
\hline SRL & $315(518)$ & 330 (669) & 0.51 & $425(566)$ & $410(726)$ & 0.72 \\
\hline SKR & 342 (796) & $327(635)$ & 0.05 & $463(900)$ & $402(685)$ & 0.84 \\
\hline SEA & 309 (607) & $334(673)$ & 0.09 & 408 (672) & $411(729)$ & 0.61 \\
\hline Valine 11 & 329 (597) & $330(742)$ & $<0.001$ & $390(633)$ & $442(833)$ & 0.47 \\
\hline $\mathrm{SE}$ & 337 (629) & $311(743)$ & $<0.001$ & $420(841)$ & 408 (673) & 0.008 \\
\hline
\end{tabular}

Table 3B. Mean autoantibody (anti-CCP) concentrations across haplotype groups and by HLA-DRB1 status.

\begin{tabular}{|c|c|c|c|c|c|c|}
\hline Haplotype & Haplotype+ & $\begin{array}{c}\text { All, } n=1441 \\
\text { Haplotype- }\end{array}$ & $\mathrm{p}^{*}$ & Haplotype+ & $\begin{array}{c}\mathrm{i}-\mathrm{CCP}+, \mathrm{n}=1 \\
\text { Haplotype- }\end{array}$ & $\mathrm{p}$ \\
\hline VKA & $331(510)$ & 243 (407) & $<0.001$ & 377 (529) & $338(445)$ & 0.09 \\
\hline VRA & 303 (449) & $261(445)$ & $<0.001$ & $360(468)$ & $350(484)$ & 0.88 \\
\hline LRA & $311(485)$ & $259(431)$ & 0.02 & $389(514)$ & 340 (466) & 0.03 \\
\hline PRA & $291(295)$ & $272(450)$ & 0.18 & $342(291)$ & $353(484)$ & 0.44 \\
\hline VRE & $267(488)$ & $273(445)$ & 0.37 & $368(542)$ & $292(357)$ & 0.60 \\
\hline DRE & $156(218)$ & $278(453)$ & 0.79 & $184(226)$ & 361 (487) & 0.003 \\
\hline VEA & 136 (164) & $274(448)$ & 0.29 & $213(160)$ & $354(481)$ & 0.76 \\
\hline SKA & 258 (304) & $273(451)$ & 0.47 & $321(308)$ & $354(485)$ & 0.44 \\
\hline PAA & $323(478)$ & $260(438)$ & 0.13 & $413(505)$ & $338(472)$ & 0.02 \\
\hline GRQ & $275(502)$ & $272(435)$ & 0.35 & $369(551)$ & $350(465)$ & 0.95 \\
\hline SRA & $244(431)$ & 277 (449) & 0.01 & 351 (479) & $353(480)$ & 0.72 \\
\hline SRE & 211 (398) & 275 (448) & 0.006 & $366(470)$ & $353(480)$ & 0.87 \\
\hline LEA & $126(167)$ & 275 (449) & 0.02 & $229(165)$ & $354(481)$ & 0.65 \\
\hline SRL & 258 (397) & 273 (449) & 0.50 & $352(426)$ & $353(482)$ & 0.90 \\
\hline SKR & 155 (336) & $296(461)$ & $<0.001$ & 259 (404) & 367 (488) & $<0.001$ \\
\hline SEA & $191(360)$ & 287 (459) & $<0.001$ & $284(408)$ & 364 (489) & 0.003 \\
\hline Valine 11 & 303 (479) & 233 (397) & $<0.001$ & $359(501)$ & $343(441)$ & 0.59 \\
\hline SE & $310(477)$ & $178(342)$ & $<0.001$ & $375(500)$ & $281(394)$ & $<0.001$ \\
\hline
\end{tabular}

Data are expressed as IU/ml, mean \pm SD. * P value by independent $\mathrm{t}$ tests after natural log transformation of autoantibody concentrations. Significant $\mathrm{p}$ values are shown in bold face. RF: rheumatoid factor; CCP: cyclic citrullinated peptides; SE: shared epitope.

$0.74-1.23)$ were significantly associated with mortality in fully adjusted models. Estimates were not substantially changed when these models were restricted to whites (data not shown). Specifically, in models restricted to whites there were no associations between valine at position 11 and all-cause mortality (HR 1.02; 95\% CI 0.81-1.29).

In fully adjusted models using an allele-based approach examining all-cause mortality, associations of DRE (HR 1.26; 95\% CI $0.74-2.15$ ) and SKA (HR 1.53; 95\% CI $0.85-2.75$ ) were not substantially altered (using PAA as the referent haplotype), although neither estimate achieved statistical significance (data not shown). The SKA haplotype (HR $2.06,95 \%$ CI $1.19-3.59$ ) was the only haplotype associated with cardiovascular mortality in standard fully adjusted models, an association that was attenuated and no longer significant using an allele-based approach (HR 1.74, 95\% CI 0.64-4.77) and PAA as the referent haplotype. No other haplotypes were associated with cardiovascular mortality in either haplotype modeling approach (Supplementary Table 5 , available with the online version of this article).

Personal non-commercial use only. The Journal of Rheumatology Copyright @ 2019 . All rights reserved. 
Table 4. Associations of HLA-DRB1 haplotypes with subcutaneous nodules and radiographic changes.

\begin{tabular}{lcc}
\hline Haplotype & Subcutaneous Nodules, OR (95\% CI) & ACR Radiographic Changes, OR (95\% CI) \\
\hline VKA & $1.03(0.81-1.32)$ & $\mathbf{1 . 3 9}(\mathbf{1 . 0 8}-\mathbf{1 . 8 0})$ \\
VRA & $1.02(0.79-1.31)$ & $0.97(0.75-1.26)$ \\
LRA & $1.16(0.90-1.50)$ & $0.96(0.73-1.25)$ \\
PRA & $1.40(0.72-2.72)$ & $1.15(0.58-2.30)$ \\
VRE & $1.14(0.62-2.10)$ & $1.81(0.91-3.64)$ \\
DRE & $1.24(0.70-2.17)$ & $1.67(0.93-2.98)$ \\
VEA & $0.73(0.19-2.81)$ & $2.11(0.50-8.82)$ \\
SKA & $1.15(0.62-2.14)$ & $1.10(0.58-2.10)$ \\
PAA & $1.01(0.76-1.35)$ & $1.29(0.96-1.72)$ \\
GRQ & $0.85(0.63-1.16)$ & $0.82(0.61-1.12)$ \\
SRA & $1.02(0.73-1.41)$ & $0.71(0.51-1.00)$ \\
SRE & $0.68(0.32-1.44)$ & $0.61(0.30-1.26)$ \\
LEA & $0.91(0.34-2.44)$ & $0.63(0.23-1.72)$ \\
SRL & $1.22(0.70-2.12)$ & $1.12(0.63-2.02)$ \\
SKR & $0.90(0.66-1.22)$ & $0.88(0.64-1.20)$ \\
SEA & $0.84(0.60-1.16)$ & $0.76(0.55-1.05)$ \\
Other genetic measures & $1.06(0.84-1.35)$ & $\mathbf{1 . 3 1}(\mathbf{1 . 0 4 - 1 . 6 7 )}$ \\
Valine at position 11 & $1.25(0.96-1.63)$ & $1.23(0.95-1.60)$ \\
Shared epitope-positive & &
\end{tabular}

OR $(95 \% \mathrm{CI})$ values adjusted for age, sex, race, smoking status, and disease duration. Significant associations are shown in bold face. ACR: American College of Rheumatology.

\section{DISCUSSION}

SE alleles have traditionally been recognized as exerting the greatest heritable influence in disease susceptibility as well as disease outcome in patients with RA. However, it has been discovered that there are at least 16 HLA-DRB1 haplotypes defined by amino acid at positions 11, 71, and 74 that together appear to better explain associations of HLA-DRB 1 with RA susceptibility and severity ${ }^{17,18}$. We aimed to externally validate these findings by assessing the associations between these 16 HLA-DRB1 haplotypes, along with SE alleles, with RA autoantibodies, extraarticular features, radiographic damage, disease activity, and all-cause mortality in US veterans with RA.

In our study, we reproduced previously published results regarding the association of amino acid position 11 on radiographic damage. Viatte, et al showed that valine at position 11 has the strongest association with erosive disease, while serine at the same position was associated with a decreased risk of erosions of the hands and feet ${ }^{17}$. Our study showed similar results that haplotypes VKA and VRE are associated with radiographic damage, while serine at amino acid position 11 (SRA and SEA) was associated with less radiographic damage. In our study, we also evaluated the association between HLA haplotypes and presence of rheumatoid nodules, which was not examined in prior studies. We did not identify a significant association between the 2 variables, possibly indicating that factors other than HLA-DRB1 genetic status are likely to influence the development of rheumatoid nodules.

We expected that valine at position 11 would be associated with increased disease activity at enrollment and over followup. Although DRE and VEA were associated with increased disease activity at enrollment, this finding did not persist with followup. It is quite possible that these associations (or lack of associations) could be affected by treatments received over time, even those predating enrollment into the registry. Indeed, the associations of VKA and haplotypes with valine at position 11 with a higher frequency of biologic use suggest that these genetic factors may indeed be associated with more severe disease earlier in the disease course, associations that are attenuated over time with the receipt of more aggressive disease-remitting treatments.

In contrast to the prior report from Viatte, et $a l^{17}$, we found neither the VKA nor SEA haplotypes to be associated with mortality. Instead, the SKA haplotype was associated with mortality. This might be explained by the differences in patient populations that were studied, with the prior report being a female-predominant cohort from the UK while our study was a male-predominant cohort of US veterans. Additionally, there were differences in smoking history $(80 \%$ ever smokers in our study), treatment, and comorbidity burden between the populations. Compared to the prior study, our study population was more frequently obese $(30 \%$ vs $21 \%$ ), older (64 vs 56 yrs), and more likely to be taking disease-modifying therapy at baseline ( $86 \%$ vs $29 \%$ ), all of which are key determinants of disease mortality ${ }^{9}$. Further, covariates in multivariable models differed between studies. We included age, sex, race, smoking status, and comorbidity burden, which can confound the associations between the haplotypes with mortality, but did not include variables associated with mortality in RA populations that could reside in the causal pathway (e.g., disease activity, functional status,

Personal non-commercial use only. The Journal of Rheumatology Copyright $@$ 2019. All rights reserved 
Associations of HLA-DRB1 Haplotypes with Mortality Adjusted for age, sex, and race

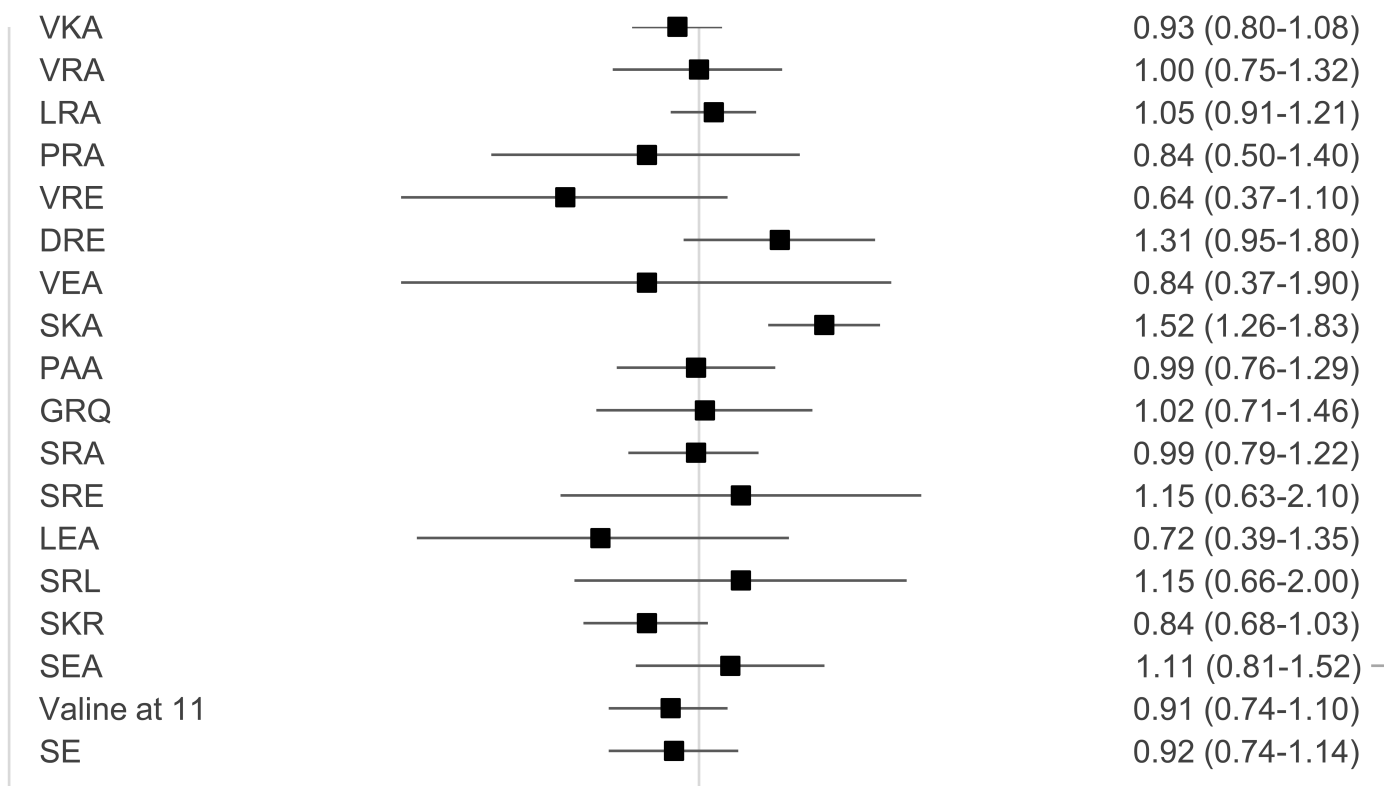

0.1

1

$\mathrm{HR}$ and $95 \% \mathrm{Cl}$

Associations of HLA-DRB1 Haplotypes with Mortality Adjusted for age, sex, race, smoking status, and RDCl

VKA
VRA
LRA
PRA
VRE
DRE
VEA
SKA
PAA
GRQ
SRA
SRE
LEA
SRL
SKR
SEA
Valine at 11
SE

0.1

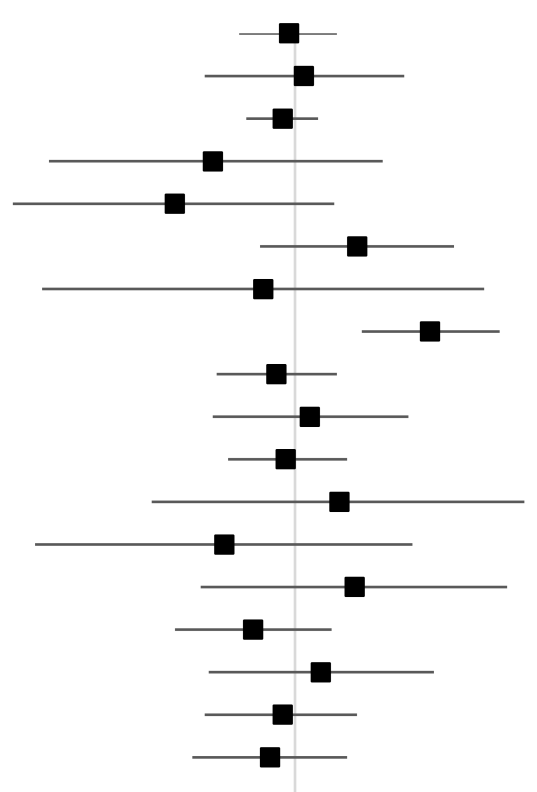

1
$0.98(0.83-1.15)$

$1.03(0.74-1.44)$

$0.96(0.85-1.08)$

$0.76(0.44-1.34)$

$0.67(0.39-1.14)$

$1.23(0.89-1.70)$

$0.90(0.43-1.88)$

1.57 (1.25-1.98)

$0.94(0.77-1.15)$

1.05 (0.76-1.46)

$0.97(0.80-1.19)$

$1.16(0.62-2.15)$

$0.79(0.42-1.48)$

$1.22(0.73-2.03)$

$0.87(0.67-1.13)$

$1.09(0.75-1.59)$

0.96 (0.74-1.23)

$0.92(0.71-1.19)$

$\mathrm{HR}$ and $95 \% \mathrm{Cl}$

Figure 1. Forest plots demonstrate associations of individual HLA-DRB1 haplotypes, haplotypes with valine at position 11, and shared epitope (SE) with all-cause mortality. Values represent HR and 95\% CI. RDCI: Rheumatic Diseases Comorbidity Index. 
medications). Similar to longitudinal disease activity ${ }^{25}$, our mortality findings suggest that other factors beyond HLA-DRB1 inheritance are the major determinants of mortality risk in patients with RA.

Our study is among the first, to our knowledge, to evaluate the associations between HLA-DRB1 haplotypes and autoantibody concentration. We found that valine at position 11 shows the strongest association with both seropositivity and autoantibody concentrations. Patients who carry haplotypes VKA, VRA, or LRA have the strongest association with seropositivity and high rates of being dual antibody positive (both RF and anti-CCP). Importantly, the frequency of haplotypes for individuals who were simultaneously negative for $\mathrm{RF}$ but positive for anti-CCP antibody was strikingly similar to the frequencies observed in patients positive for both autoantibodies, suggesting that HLA-DRB1 associations with seropositivity are driven primarily by anti-CCP. The valinecontaining VKA haplotype is associated with higher titer of both RF and anti-CCP, and haplotype VRA was associated with high anti-CCP titer. However, when stratified by smoking status, only the associations with anti-CCP persisted. Serine at the same position appears to be protective of autoantibody development, given observed associations of relevant haplotypes with lower titers of RF (SKE) and anti-CCP (SRA, SRE, SKR, and SEA). Given the previously demonstrated associations between autoantibodies and disease severity ${ }^{19}$, this result may help explain the association between valine at position 11 on greater disease activity and radiographic damage as well as the protective effect of serine at position 11 on radiographic damage.

Given the male predominance and frequent smoking history, our results may not generalize to other RA populations. However, the unique cohort characteristics provide the opportunity for external validity testing in a heterogeneous RA population. Though assessed in prior reports, we did not evaluate the associations between HLA-DRB1 haplotypes and treatment response, a question that, while important, cannot be adequately addressed with this observational cohort. Likewise, our available data precluded us from examining genetic associations with the development of rheumatoid nodules over time or radiographic progression. Strengths of our study include the broad range of outcomes evaluated such as standardized autoantibodies (with their concentrations) measured in a central laboratory, clinical manifestations, longitudinal disease activity, and mortality.

Our study showed HLA-DRB1 haplotype status is an important independent predictor of seropositivity, autoantibody concentrations, disease activity, and outcome in RA. Valine at amino acid position 11 is associated with RA autoantibodies and radiographic damage, while serine at the same position is associated with a lower titer of autoantibodies. However, we did not replicate findings linking the VKA haplotype and valine at position 11 to increased mortality risk.

\section{ACKNOWLEDGMENT}

Work supported by the US Center of Excellence for Suicide Prevention, Joint Department of Veterans Affairs and Department of Defense Suicide Data Repository - National Death Index.

\section{ONLINE SUPPLEMENT}

Supplementary material accompanies the online version of this article.

\section{REFERENCES}

1. Holoshitz J. The rheumatoid arthritis HLA-DRB1 shared epitope. Curr Opin Rheumatol 2010;22:293-8.

2. Gonzalez-Gay MA, Garcia-Porrua C, Hajeer AH. Influence of human leukocyte antigen-DRB1 on the susceptibility and severity of rheumatoid arthritis. Semin Arthritis Rheum 2002;31:355-60.

3. Mattey DL, Thomson W, Ollier WE, Batley M, Davies PG, Gough AK, et al. Association of DRB1 shared epitope genotypes with early mortality in rheumatoid arthritis: results of eighteen years of followup from the early rheumatoid arthritis study. Arthritis Rheum 2007;56:1408-16.

4. van Gaalen FA, van Aken J, Huizinga TW, Schreuder GM, Breedveld FC, Zanelli E, et al. Association between HLA class II genes and autoantibodies to cyclic citrullinated peptides (CCPS) influences the severity of rheumatoid arthritis. Arthritis Rheum 2004;50:2113-21.

5. Weyand CM, Xie C, Goronzy JJ. Homozygosity for the HLA-DRB1 allele selects for extraarticular manifestations in rheumatoid arthritis. J Clin Invest 1992;89:2033-9.

6. Farragher TM, Goodson NJ, Naseem H, Silman AJ, Thomson W, Symmons D, et al. Association of the HLA-DRB1 gene with premature death, particularly from cardiovascular disease, in patients with rheumatoid arthritis and inflammatory polyarthritis. Arthritis Rheum 2008;58:359-69.

7. Gonzalez-Gay MA, Gonzalez-Juanatey C, Lopez-Diaz MJ, Pineiro A, Garcia-Porrua C, Miranda-Filloy JA, et al. HLA-DRB1 and persistent chronic inflammation contribute to cardiovascular events and cardiovascular mortality in patients with rheumatoid arthritis. Arthritis Rheum 2007;57:125-32.

8. Birru Talabi M, Mackey RH, Kuller LH, Dorman JS, Deane KD, Robinson WH, et al. Human leukocyte antigen shared epitope and inflammation, cardiovascular disease, cancer, and mortality among postmenopausal women in the women's health initiative rheumatoid arthritis study. Am J Epidemiol 2017;186:245-54.

9. England BR, Sayles H, Michaud K, Caplan L, Davis LA, Cannon $\mathrm{GW}$, et al. Cause-specific mortality in male us veterans with rheumatoid arthritis. Arthritis Care Res 2016;68:36-45.

10. Balsa A, Cabezon A, Orozco G, Cobo T, Miranda-Carus E, Lopez-Nevot MA, et al. Influence of HLA DRB1 alleles in the susceptibility of rheumatoid arthritis and the regulation of antibodies against citrullinated proteins and rheumatoid factor. Arthritis Res Ther 2010;12:R62.

11. Chun-Lai T, Padyukov L, Dhaliwal JS, Lundstrom E, Yahya A, Muhamad NA, et al. Shared epitope alleles remain a risk factor for anti-citrullinated proteins antibody (ACPA) - positive rheumatoid arthritis in three Asian ethnic groups. PLoS One 2011;6:e21069.

12. Schett $\mathrm{G}$, Gravallese E. Bone erosion in rheumatoid arthritis: mechanisms, diagnosis and treatment. Nat Rev Rheumatol 2012;8:656-64.

13. Hedger SC, Macardle P, Bond MJ, Ahern MJ, Smith MD, Roberts-Thomson PJ. Shared rheumatoid epitope as a risk factor in determining outcome in rheumatoid arthritis. Aust $\mathrm{N} Z \mathrm{~J}$ Med 1999;29:234-8.

14. Gorman JD, Lum RF, Chen JJ, Suarez-Almazor ME, Thomson G, Criswell LA. Impact of shared epitope genotype and ethnicity on erosive disease: a meta-analysis of 3,240 rheumatoid arthritis patients. Arthritis Rheum 2004;50:400-12. 
15. Turesson C, Schaid DJ, Weyand CM, Jacobsson LT, Goronzy JJ, Petersson IF, et al. The impact of HLA-DRB1 genes on extra-articular disease manifestations in rheumatoid arthritis. Arthritis Res Ther 2005;7:R1386-93.

16. Gorman JD, David-Vaudey E, Pai M, Lum RF, Criswell LA. Lack of association of the HLA-DRB1 shared epitope with rheumatoid nodules: an individual patient data meta-analysis of 3,272 Caucasian patients with rheumatoid arthritis. Arthritis Rheum 2004;50:753-62.

17. Viatte S, Plant D, Han B, Fu B, Yarwood A, Thomson W, et al. Association of HLA-DRB1 haplotypes with rheumatoid arthritis severity, mortality, and treatment response. JAMA 2015; 313:1645-56

18. Raychaudhuri S, Sandor C, Stahl EA, Freudenberg J, Lee HS, Jia X, et al. Five amino acids in three HLA proteins explain most of the association between MHC and seropositive rheumatoid arthritis. Nat Genet 2012;44:291-6.

19. Ling SF, Viatte S, Lunt M, Van Sijl AM, Silva-Fernandez L, Symmons DP, et al. HLA-DRB1 amino acid positions 11/13, 71, and 74 are associated with inflammation level, disease activity, and the health assessment questionnaire score in patients with inflammatory polyarthritis. Arthritis Rheumatol 2016;68:2618-28.

20. Mikuls TR, Fay BT, Michaud K, Sayles H, Thiele GM, Caplan L, et al. Associations of disease activity and treatments with mortality in men with rheumatoid arthritis: results from the VARA registry. Rheumatology 2011;50:101-9.

21. Arnett FC, Edworthy SM, Bloch DA, McShane DJ, Fries JF, Cooper NS, et al. The American Rheumatism Association 1987 revised criteria for the classification of rheumatoid arthritis. Arthritis Rheum 1988;31:315-24.

22. England BR, Sayles H, Mikuls TR, Johnson DS, Michaud K. Validation of the rheumatic disease comorbidity index. Arthritis Care Res 2015;67:865-72.

23. Miriovsky BJ, Michaud K, Thiele GM, O’Dell JR, Cannon GW, Kerr G, et al. Anti-CCP antibody and rheumatoid factor concentrations predict greater disease activity in men with rheumatoid arthritis. Ann Rheum Dis 2010;69:1292-7.

24. Mikuls TR, Gould KA, Bynote KK, Yu F, Levan TD, Thiele GM, et al. Anticitrullinated protein antibody (ACPA) in rheumatoid arthritis: influence of an interaction between HLA-DRB1 shared epitope and a deletion polymorphism in glutathione s-transferase in a cross-sectional study. Arthritis Res Ther 2010;12:R213.

25. Schisterman EF, Cole SR, Platt RW. Overadjustment bias and unnecessary adjustment in epidemiologic studies. Epidemiology 2009;20:488-95. 